\title{
Inklusion von Menschen ohne Internet in zufallsbasierte Onlinepanel-Umfragen
}

Carina Cornesse, Universität Mannheim

Ines Schaurer, GESIS - Leibniz Institut für Sozialwissenschaften

\section{Einleitung}

Zur Beantwortung vieler Forschungsfragen in den Sozialwissenschaften ist es unerlässlich Umfragen durchzuführen, mittels derer die Forschenden Einblicke in die Einstellungen und das Verhalten der Gesamtbevölkerung erlangen. Um zu gewährleisten, dass die aus den Umfragedaten generierten Erkenntnisse auf die Gesamtbevölkerung übertragbar sind, werden klassischerweise zufallsbasierte Stichproben gezogen, beispielsweise aus Bevölkerungsregistern. Die in die Stichprobe gezogenen Personen oder Haushalte werden daraufhin anhand der aus dem Stichprobenrahmen verfügbaren Informationen kontaktiert und befragt. Die Befragung findet in der Regel offline statt, also zum Beispiel per persönlichem oder telefonischem Interview. Beispiele für etablierte deutsche Studien, die auf diese Weise durchgeführt werden sind die Allgemeine Bevölkerungsumfrage der Sozialwissenschaften (ALLBUS, siehe Wasmer et al., 2017) und das Sozioökonomische Panel (SOEP, siehe Pagel und Schupp, 2019).

Im Gegensatz zu solchen klassischen Umfragedesigns werden sozialwissenschaftliche Umfragen seit dem Beginn des 21. Jahrhunderts zunehmend mittels Onlinepanels im Internet durchgeführt (für einen Überblick siehe Callegaro et al., 2014). Während Onlinepanels meist eine enorme Kosten- und Zeitersparnis darstellen, beruhen sie herkömmlicherweise auf nichtzufallsbasierten Stichproben, die nachgewiesenermaßen keine zuverlässigen Rückschlüsse auf die Gesamtbevölkerung zulassen (für einen Überblick zum Thema zufallsbasierte und nichtzufallsbasierte Stichproben siehe Cornesse et al., 2020). 
Nur eine vergleichsweise kleine Anzahl von Studien versucht die Vorteile der klassischen Umfragedesigns, die zuverlässige Rückschlüsse auf die Gesamtbevölkerung zulassen, mit den Vorteilen der aufgekommenen Onlinepanels zu verbinden, die schnell, flexibel, und kostensparend agieren können (zur praktischen Relevanz dieser Vorteile für die Wissenschaft siehe z.B. Blom et al., 2020 und Schaurer und Weiß, 2020).

Solche Studien werden als zufallsbasierte Onlinepanels bezeichnet und basieren, wie die klassischen sozialwissenschaftlichen Umfragen, auf Zufallsstichproben, die beispielsweise aus Bevölkerungsregistern gezogen werden. Ebenfalls wie in den klassischen sozialwissenschaftlichen Umfragen nutzen die probabilistischen Onlinepanels die Kontaktinformationen aus dem Stichprobenrahmen (z.B. Adressdaten) um die gezogenen Stichprobenmitglieder zu kontaktieren. Spätestens nach einem meist kurzen Rekrutierungsinterview werden die Stichprobenmitglieder dann allerdings gebeten per Internet an Folgebefragungen teilzunehmen (siehe DiSogra \& Callegaro, 2016). Alle Personen, die diesen Folgebefragungen zustimmen und sich online dazu registrieren, gelten dann als rekrutierte Onlinepanel-Mitglieder. Beispiele für zufallsbasierte Onlinepanels, die in Deutschland durchgeführt werden, sind das German Internet Panel (GIP, siehe Blom et al., 2015) und das GESIS Panel (siehe Bosnjak et al., 2018).

Der in der Rekrutierung von zufallsbasierten Onlinepanels notwendige Wechsel von einem initialen Kontaktmodus (persönlich, telefonisch, oder postalisch) zum eigentlichen Studienmodus im Internet birgt einige Herausforderungen für das Studiendesign. Im Vordergrund steht dabei besonders die Problematik, dass nicht alle Menschen das Internet nutzen. Insofern sich Personen, die das Internet nutzen, systematisch von Personen unterscheiden, die das Internet nicht nutzen, kann dies potentiell zu falschen Rückschlüssen aus den Umfragedaten der Onlinepanels auf die Allgemeinbevölkerung führen. Im vorliegenden Beitrag soll es daher darum gehen die Problematik der Exklusion von Menschen ohne Internet 
zu beschreiben und die in der Praxis verwendeten Lösungsansätze der Inklusion von Menschen ohne Internet in zufallsbasierten Onlinepanels zu diskutieren.

\section{Menschen ohne Internet in der Bevölkerung}

Es ist ein international bekanntes Phänomen, dass Teile der Bevölkerung das Internet nicht nutzen (siehe z.B. Statista, 2020). In Deutschland lag dieser Bevölkerungsanteil der Menschen, die das Internet nicht nutzen 2013 bei 21\% und ist seitdem auf 12\% im Jahr 2019 gesunken (Destatis, 2020). In der Europäischen Union insgesamt liegt der Anteil der Menschen die das Internet nicht nutzen bei $11 \%$ (siehe Internet World Stats, 2019).

Die Gründe warum manche Menschen das Internet nicht nutzen sind vielfältig. Laut einer Studie von Eynon und Helsper (2011) können vier Gruppen von Gründen der NichtInternetnutzung identifiziert werden: Kosten, Interesse, Kenntnisse und Zugang. Kostengründe beziehen sich vorrangig auf die finanziellen Aufwendungen, die aufgebracht werden müssen um eine Internetnutzung zu gewährleisten. Unter diese finanziellen Kosten fallen sowohl die Aufwendungen für ein internetfähiges Gerät, wie zum Beispiel einen PC und/oder ein Smartphone, als auch die Aufwendungen für Datennutzungsverträge und/ oder Prepaid-Karten. Hinzu kommen weiterhin häufig zusätzliche Ausstattungsgegenstände, wie Router oder WLAN-Sticks, sowie möglicherweise notwendige Aufwendungen für die Instandhaltung und/oder Reparatur der Ausstattungsgegenstände.

Während Kostengründe sich auf externe, vorrangig finanzielle, Faktoren beziehen, die sich außerhalb der Kontrolle der betroffenen Individuen befinden können, beziehen sich Interessensgründe der (Nicht-)Internetnutzung auf den Lebenszusammenhang der Menschen und ihre daraus resultierende Motivation, sich mit dem Internet zu beschäftigen. Wenn ein Mensch sich zum Beispiel in einem sozialen Umfeld bewegt, in dem die relevanten Anderen ebenfalls nicht das Internet nutzen und in dem daher die Notwendigkeit und der Nutzen des Internets als begrenzt empfunden werden, mag das zu mangelndem Interesse am Internet führen 
und, in der Konsequenz dazu, dass uninteressierte Menschen dann das Internet tatsächlich nicht nutzen.

Kenntnisgründe der (Nicht-)Internetnutzung wiederum beziehen sich auf die Notwendigkeit, sich bestimmte Fertigkeiten und spezialisiertes Wissen anzueignen. Dies beinhaltet zum Beispiel das Wissen darum wie man eine Tastatur, einen Touchscreen oder eine Maus verwendet und die Kenntnis darum wie man einen Web-Browser oder eine Suchmaschine bedient. In dem Sinne wie die Aneignung dieser Kenntnisse Zeitressourcen verbraucht und möglicherweise sogar Schulungsgebühren notwendig macht, können die Kenntnisgründe auch im weitesten Sinne als (Opportunitäts-) Kosten verstanden werden, die umso höher sind, je weniger eine Person bereits in eine Umgebung hinein-sozialisiert wurde, in der technische Geräte und das Internet schon immer zum Alltag gehört haben (z.B. sogenannte „digital natives“, siehe Prensky, 2001).

Zugangsgründe hingegen beziehen sich auf die Problematik, dass, selbst wenn die diversen Kosten getragen werden können, das Interesse am Internet vorhanden ist und die notwendigen Kenntnisse bestehen, strukturell bedingt kein oder nur unzureichender Internetzugang verfügbar sein kann. In Deutschland ist die Versorgung mit schnellem Breitbandinternet zum Beispiel regional unterschiedlich stark ausgebaut. So liegt die Breitbandversorgung in und um Großstädte wie Berlin und München sowie in großen Ballungszentren wie dem Rhein-MainGebiet oder dem Ruhrgebiet meist bei über 95\% der Privathaushalte (Bundesministerium für Verkehr und digitale Infrastruktur, 2020). In ländlichen Regionen dagegen, wie zum Beispiel in Nordhessen, und in weiten Teilen Ostdeutschlands, insbesondere MecklenburgVorpommern, liegt die Breitbandversorgung häufig bei deutlich unter 50\% der Privathaushalte (sowohl leitungsgebunden als auch mobil).

Insgesamt sind Zugangsgründe der Nicht-Internetnutzung diejenigen, die am stärksten außerhalb der Kontrolle der betroffenen Individuen liegen, gefolgt von Kostengründen, die sich 
in besonderem Maße auf die finanziellen Ressourcen von Personen beziehen. Interessengründe und Kenntnisgründe dagegen können von den betroffenen Personen kontrolliert werden, auch wenn sie von sozialen Kontexten geprägt werden und dadurch ungleich verteilte Startbedingungen in der Bevölkerung hervorrufen (insbesondere im Hinblick auf sogenannte „digital skills“, siehe z.B. Van Laar et al., 2017).

Aus all dem ergibt sich die Problematik zufallsbasierter Onlinepanels, dass diejenigen Menschen in der Bevölkerung, die das Internet nicht nutzen, sich systematisch von denjenigen Menschen unterscheiden können, die das Internet nutzen. Eine Exklusion der NichtInternetnutzenden könnte somit dazu führen, dass die Befragtenstichproben nicht ausreichend repräsentativ sind, um Rückschlüsse auf die Gesamtbevölkerung zu erlauben. Dabei spielen nicht nur geographische Indikatoren (z.B. Ost versus West und Stadt versus Land, siehe Bundesministerium für Verkehr und digitale Infrastruktur, 2020) eine Rolle, sondern auch andere sozio-demographische Faktoren. In einem internationalen Kontext, beispielsweise, zeigen Helsper und Reisdorf (2017), dass Menschen ohne Internet im Durchschnitt älter sind, geringer gebildet, und mit einer höheren Wahrscheinlichkeit arbeitslos als Menschen mit Internet. Diese Erkenntnisse sind auf den deutschen Kontext übertragbar (siehe z.B. Bosnjak et al., 2013, König et al., 2018 und Schleife, 2010).

\section{Inklusionsstrategien zufallsbasierter Onlinepanels}

Da die Exklusion von Menschen ohne Internet ein potentielles Problem für die Stichprobenqualität von Onlinepanels darstellt, soll es im folgenden Abschnitt darum gehen, welche Inklusionsstrategien bestehende Onlinepanels verwenden, um dieser Gefahr entgegenzuwirken. Grundsätzlich kann man zwei Strategien zur Inklusion von Menschen ohne Internet unterscheiden: die Ausstattung der Personen mit entsprechender Ausrüstung zur Teilnahme an Befragungen per Internet und die Befragung mittels eines alternativen Teilnahmemodus außerhalb des Internets. Tabelle 1 bietet einen Überblick über gängige 
Strategien zur Inklusion von Menschen ohne Internet, inklusive einer beispielhaften Übersicht in welchen Onlinepanels diese Strategien zum Einsatz kommen.

Tabelle 1. Strategien zur Inklusion von Menschen ohne Internet und Überblick gängiger zufallsbasierter Onlinepanels, die solche Strategien verwenden.

\begin{tabular}{lll}
\hline Inklusionsstrategie & Panel & Land \\
\hline & American Trends Panel (nach 2016) & USA \\
$\begin{array}{l}\text { Ausstattung mit } \\
\text { Ausrüstungsgegenständen } \\
\text { (z.B. WLAN und }\end{array}$ & GLIPSS Panel & Frankreich \\
$\begin{array}{l}\text { PC/Tablet) } \\
\text { Ipsos Knowledge Panel }\end{array}$ & Deutschland \\
& LISS Panel & USA \\
\hline $\begin{array}{l}\text { Postalische Befragung als } \\
\text { alternativer } \\
\text { Teilnahmemodus }\end{array}$ & American Trends Panel (bis 2016) & USA \\
\hline $\begin{array}{l}\text { Telefonische Befragung } \\
\text { als alternativer } \\
\text { Teilnahmemodus }\end{array}$ & GESIS Panel & Deutschland \\
\hline $\begin{array}{l}\text { Teilnahmemodi } \\
\text { Tehrere alternative }\end{array}$ & NatCen Panel & USA \\
\hline & Probit & Großbritannien \\
\hline
\end{tabular}

Eine vergleichsweise häufig verwendete Strategie zur Inklusion von Menschen ohne Internet in ein zufallsbasiertes Onlinepanel ist die Ausstattung der Personen mit Ausrüstungsgegenständen. Diese Inklusions-Strategie wird in Deutschland beispielsweise vom GIP verwendet (siehe Blom et al., 2017, für aktuelle Informationen zum GIP siehe auch Universität Mannheim, 2020) und ist auch im internationalen Kontext verbreitet (z.B. American Trends Panel, ELIPSS Panel, Ipsos Knowledge Panel, LISS Panel, Understanding America 
Panel; siehe Pew Research Center, 2019, Ipsos, 2020; CentERdata, 2020; University of Southern California, 2017).

Die Strategie der Ausstattung von Menschen ohne Internet erfolgt zumeist indem die gezogenen Stichprobenmitglieder im Prozess der Panel-Rekrutierung nach ihrer Ausstattung mit Internet am Wohnort gefragt werden. Personen, die angeben nicht über eine Internetverbindung und/oder ein internet-fähiges Endgerät zu verfügen, werden dann mit der entsprechenden Ausrüstung ausgestattet (siehe z.B. Blom et al., 2017). Zumeist sieht diese Ausrüstung die Bereitstellung eines WLAN-Routers sowie eines PCs oder Tablets am Wohnort vor (siehe z.B. Pew Research Center, 2019).

Insgesamt zielt die Ausstattung von Menschen ohne Internet mit den notwendigen Ausrüstungsgegenständen darauf ab, die Kostengründe zu beseitigen, die Menschen von der Nutzung des Internets abhalten. Insofern auch die Installation der Geräte von externen Dienstleistern übernommen und sogar Schulungen angeboten werden, bemühen sich solche Strategien auch um eine Verringerung der Kenntnisgründe, aus denen manche Menschen das Internet nicht nutzen (siehe z.B. Blom et al., 2017). Insbesondere die Interessensgründe und die Zugangsgründe der Nicht-Internetnutzung können von Ausstattungsstrategien allerdings nur begrenzt adressiert werden.

Während einige zufallsbasierten Onlinepanels die Ausstattungsstrategie der Inklusion von Menschen ohne Internet nutzen, haben andere zufallsbasierte Onlinepanels eine mixed-mode Strategie etabliert, in der Menschen, die das Internet nicht nutzen mittels eines alternativen Befragungsmodus an den Panel-Befragungen teilnehmen können. Die in der Praxis verwendeten alternativen Befragungsmodi sind die postalische Befragung, die in Deutschland zum Beispiel im GESIS Panel (Bosnjak et al., 2018) Anwendung findet und die in den USA außerdem bis 2016 im American Trends Panel verfolgt wurde (siehe Pew Research Center, 2019), sowie die telefonische Befragung, die zum Beispiel im AmeriSpeak Panel (NORC, 
2019), NatCen Panel (NatCen Social Research, 2020) und Probit Panel (Probit Inc., 2020) verwendet wird. Teilweise bieten Onlinepanels auch mehrere alternative Befagungsmodi an, wie zum Beispiel das Gallup Panel (GALLUP, 2020) und KAMOS (Center for Asian Public Opinion Research \& Collaboration Initiative, 2020).

Die Strategie des alternativen Befragungsmodus erfolgt zumeist indem die gezogenen Stichprobenmitglieder im Prozess der Panel-Rekrutierung nach ihrer privaten Internetnutzung befragt werden. Personen, die angeben das Internet nicht zu nutzen, wird dann der alternative Panel-Teilnahmemodus angeboten (siehe z.B. NORC, 2019). Teilweise wird der alternative Teilnahmemodus in diesem Prozess auch solchen Personen angeboten, die zwar über die notwendige Ausstattung zur Teilnahme am Panel per Internet verfügen, diese Ausstattung aber nicht zur Panel-Teilnahme nutzen möchten (siehe Bosnjak et al., 2018).

Während die Ausstattungsstrategie zur Inklusion von Menschen ohne Internet ihren Schwerpunkt darauf hat die Gründe der Nicht-Internetnutzung zu beseitigen, fokussiert die Strategie des Anbietens eines alternativen Modus darauf diese Barrieren zu umgehen, indem sie die Internetnutzung für die Panelteilnahme entbehrlich macht. Damit ist es für die am Panel teilnehmende Person weder notwendig die Kosten der Internutzung tragen zu können, noch die notwendigen technologischen Kenntnisse oder entsprechendes Interesse an der Internetnutzung zu haben. Auch die Problematik des potentiellen strukturellen Mangels an Zugang zum Internet kann damit umgangen werden.

Dadurch, dass die Strategie des alternativen Teilnahme-Modus alle Internet-Nutzungsbarrieren umgeht stellt sie für das zu rekrutierende Stichprobenmitglied eine geringe Teilnahme-Hürde dar: Es ist nicht notwendig sich mit technischer Ausstattung auseinanderzusetzen, neue Kenntnisse zu erarbeiten und die entsprechende Motivation aufzubringen, sich auf etwas Neues einzulassen. Die reduzierte initiale Teilnahme-Hürde, die die alternative Modus-Strategie bietet, führt allerdings auch zu einer Begrenzung der Befragungsmöglichkeiten im regulären 
Verlauf des Panels. So ist, zum Beispiel, insbesondere im postalischen Befragungsmodus, keine Einbindung von Audio- und Videomaterial in die Panel-Befragungen möglich und die Möglichkeiten zur Implementierung komplexer Fragebogenexperimente und Filterführungen ist stark begrenzt. Außerdem muss meist eine längere Feldphase für die Panel-Befragungen eingeräumt werden, weil beispielsweise die Dauer der Postwege berücksichtigt werden muss. Hinzu kommt die mögliche Problematik von Modus-Effekten in den Befragungsdaten (zu Modus-Effekten in mixed-mode Umfragen siehe z.B. Vannieuwenhuyze und Loosveldt, 2013), insbesondere wenn der selbst-administrierte Befragungsmodus per Internet mit einem interviewer-administrierten Befragungsmodus kombiniert wird (zu Modus-Effekten zwischen interviewer-administrierten und selbst-administrierten Befragungen siehe z.B. Heerwegh, 2009).

Die Ausstattungsstrategie dagegen profitiert im regulären Panelbetrieb davon, dass alle am Panel teilnehmenden Personen in demselben Modus per Internet befragt werden. Dadurch werden die in mixed-mode-Befragungen möglichen Modus-Effekte umgangen, Feldphasen können kurzgehalten werden (zu den Vorteilen davon siehe z.B. Blom et al., 2020), und das volle Potential der technischen Möglichkeiten kann ausgeschöpft werden. Der Preis dafür ist die vergleichsweise hohe Hürde von Personen ohne Internet im Panel-Rekrutierungsprozess, der zwar durch Schulungsangebote minimiert werden kann, potentiell aber trotzdem zu einer geringen Panel-Rekrutierungswahrscheinlichkeit unter den Stichprobenmitgliedern ohne Internet führen kann. Diese erhöhte Rekrutierungshürde kann dazu führen, dass nicht genügend Menschen ohne Internet in das Onlinepanel rekrutiert werden können um einen positiven Effekt der Inklusionsstrategie auf die Paneldaten erzielen zu können.

\section{Erkenntnisse zur Inklusion von Menschen ohne Internet in Onlinepanels}

Während es einige zufallsbasierte Onlinepanels gibt, die Strategien zur Inklusion von Menschen ohne Internet nutzen, sind bisher vergleichsweise wenige Erkenntnisse zu deren 
Erfolg publiziert worden. Die meisten publizierten Artikel zu dem Thema beschäftigen sich mit dem Erfolg einer einzelnen Strategie in einem bestimmten Panel zum Zeitpunkt der PanelRekrutierung. Dabei werden häufig die Rekrutierungsraten zwischen Menschen mit und ohne Internet in das jeweilige Panel verglichen. Zusätzlich werden meist deskriptive Analysen zu der Frage präsentiert, ob die Inklusion von Menschen ohne Internet einen positiven Einfluss auf die Stichprobenqualität des frisch rekrutierten Panels hat. Es bestehen daher untere anderem Forschungslücken im Bereich des Vergleichs mehrerer Strategien zur Inklusion von Menschen ohne Internet sowie Analysen zum Erfolg der Inklusionsstrategien im regulären Panelbetrieb (d.h. nach der Rekrutierung des Panels).

Wir stellen hier daher zunächst den Stand der publizierten Literatur zum Thema Inklusion dar, bevor wir einige zusätzliche Ergebnisse aus dem German Internet Panel (GIP) und GESIS Panel präsentieren, die zur Schließung der genannten Forschungslücken beitragen.

\section{Erkenntnisse aus der Literatur: Rekrutierungswahrscheinlichkeit}

Ein wichtiges Kriterium zur Bewertung der existierenden Inklusionsstrategien ist die Frage, ob es den Onlinepanels gelingt Menschen ohne Internet in das Panel zu rekrutieren. Im Idealfall sollte die Rekrutierungsrate von Menschen ohne Internet vergleichbar hoch sein wie die Rekrutierungsrate von Menschen mit Internet.

Entgegen diesem Ideal finden einige Studien, dass die Rekrutierungsrate von Menschen ohne Internet unter der Rekrutierungsrate von Menschen mit Internet liegt. So finden Blom, Gathmann und Krieger (2015), dass die Rekrutierungsrate von Menschen ohne Internet zum GIP rund 35\%-Punkte unter der von Menschen mit Internet liegt. Leenheer und Scherpenzeel (2013) kommen zu einem ähnlich klaren Ergebnis in Bezug auf das niederländische LISS Panel, mit einer Rekrutierungsrate von Menschen ohne Internet, die 49\%-Punkte unter der Rekrutierungsrate von Menschen mit Internet liegt. Ebenso klare Differenzen wurden von Revilla et al. (2016) aus dem französischen ELIPSS Panel berichtet, mit Rekrutierungsraten 
von Menschen ohne Internet, die bis zu knapp 80\%-Punkte unter denen von Menschen mit Internet liegen. Es zeigt sich also insgesamt, dass die genannten Studien eine geringere Rekrutierungswahrscheinlichkeit von Nicht-Internetnutzenden im Vergleich zu Internetnutzenden berichten.

Darüber, ob und wie sich das Teilnahmeverhalten von Menschen mit und ohne Internet nach der Panel-Rekrutierung, also im regulären Panelbetrieb unterscheidet, ist kaum etwas aus der publizierten Literatur bekannt. Eine der wenigen Studien, die sich mit dieser Thematik beschäftigt, stammt von Toepoel und Hendriks (2016). In dieser Studie wird berichtet, dass Menschen, die im LISS Panel mit Internetzugang und internetfähigen Geräten ausgestattet wurden, im regulären Panelbetrieb eine höhere Wahrscheinlichkeit zur Teilnahme an den PanelWellen aufweisen. Dies legt die Vermutung nahe, dass solche Stichprobenmitglieder, die die erhöhten Opportunitätskosten der Ausstattung im Rekrutierungsprozess auf sich genommen haben, dann auch den vollen Nutzen der Panelteilnahme für sich in Anspruch nehmen (z.B. die Möglichkeit, ihre Meinungen durch die Panelbefragungen mitzuteilen oder die häufig verwendeten finanzielle Belohnung für das Ausfüllen der Fragebögen zu erhalten). Im Kontrast dazu berichtet Jessop (2017) eine geringere Teilnahmewahrscheinlichkeit für die Befragten des britischen NatCen Panels, die nicht online teilnehmen. Im Gegensatz zu den Befragten des LISS Panels werden die NatCen Panel-Befragten nicht ausgestattet, sondern telefonisch kontaktiert. Dies legt die Vermutung nahe, dass die Inklusionsstrategien per Ausstattung und die Inklusionsstrategien per alternativem Befragungsmodus unterschiedliche Effekte auf das Teilnahme-Verhalten der inkludierten Menschen im Onlinepanel haben.

\section{Erkenntnisse aus der Literatur: Stichprobenqualität}

Ebenso, wie in der publizierten Literatur nur wenige Erkenntnisse über die Rekrutierungs- und Teilnahmewahrscheinlichkeit von Menschen mit und ohne Internet verfügbar sind, so gilt Selbiges auch für die Frage ob Inklusionsstrategien sich positiv auf die Stichprobenqualität 
eines Panels auswirken. Im Idealfall führt die Inklusion von Menschen ohne Internet dazu, dass Verzerrungen im Panel ausgeglichen werden, zum Beispiel indem die Inklusionsstrategien den Anteil älterer Menschen steigern, die häufig weniger Internet-affin sind (siehe z.B. Seifert und Schelling, 2018). Auch für die Literatur zur Stichprobenqualität gilt, dass die existierenden Studien auf den Einfluss der Inklusionsstrategien im Panel-Rekrutierungsprozess fokussieren und nicht auf den regulären Panelbetrieb nach der Rekrutierung.

Während die wenigen Studien zur Rekrutierungswahrscheinlichkeit darauf hindeuten, dass Menschen ohne Internet in geringerem Maße zu Onlinepanels rekrutiert werden können als Menschen mit Internet, so ist das Bild, das sich im Hinblick auf den Einfluss der Inklusion auf die Stichprobenqualität der Panels ergibt, gemischter. So finden zum Beispiel Blom et al. (2017) im GIP und Leenheer und Scherpenzeel (2013) im LISS Panel einen positiven Einfluss der Inklusion von Menschen ohne Internet auf die Panel-Stichprobenqualität im Hinblick auf Alter und Haushaltsgröße. Bosnjak et al. (2018) finden hingegen keinen statistisch signifikanten positiven Effekt im Hinblick auf diese Merkmale im GESIS Panel. Rookey, Hanway und Dillman (2008) finden im amerikanischen Gallup Panel sogar einen negativen Einfluss der Inklusion von Menschen ohne Internet auf die Stichprobenqualität im Hinblick auf das Alter.

Die unterschiedlichen Ergebnisse der Studien mögen länderbedingt sein (z.B. unterschiedliche Effekte in Europa verglichen mit den USA), zeitbedingt (z.B. unterschiedliche Effekte in den frühen Studien, als das Internet noch nicht so stark in der Bevölkerung verbreitet war, verglichen mit späteren Studien) oder auch strategiebedingt (d.h. unterschiedliche Effekte in Panels, die die Internet-Ausstattungsstrategie verwenden als in Panels, die die Strategie des alternativen Befragungsmodus verfolgen). Insgesamt ist die Studienlage noch nicht ausreichend um diese wichtige Frage zu klären.

Das einzige Merkmal bei dem alle Studien, die es untersucht haben, einen positiven Einfluss der Inklusionsstrategien auf die Panel-Stichprobenqualität finden, ist die Bildung (Blom et al., 
2017; Bosnjak et al., 2018; Rookey et al., 2008). Hier zeigt sich durchgängig, dass die Inklusion von Menschen ohne Internet die Unterrepräsentierung gering Gebildeter statistisch signifikant reduziert.

Während alle Studien zum Einfluss der Inklusion von Menschen ohne Internet auf die Onlinepanel-Stichprobenqualität zu dem Schluss kommen, dass dieser Einfluss auf mindestens einem (wenn nicht sogar allen) untersuchten Merkmalen positiv ist, so gibt es jedoch auch Forschungsergebnisse, die den Nutzen dieses positiven Effekts für inhaltliche Analysen in den Zweifel ziehen. So finden Eckman (2016) und Toepoel und Hendriks (2016) für das LISS Panel, dass multivariate Analysen zu unterschiedlichen Themen (z.B. Familie, Arbeit, Gesundheit und Politik) zu denselben Schlussfolgerungen führen unabhängig davon, ob die mit Geräten ausgestatteten Personen in die Analyse inkludiert werden oder aber aus der Analyse exkludiert werden. Außerhalb des LISS Panels ist bisher unseres Wissens nur eine Studie veröffentlicht worden, die sich mit dem Einfluss der Inklusion von Menschen ohne Internet auf inhaltliche Analyseergebnisse beschäftigt. Diese Studie wurde von Pforr und Dannwolf (2017) mit Daten des GESIS Panels durchgeführt und kommt zu dem Schluss, dass univariate Schätzungen zu politischen Einstellungen zu unterschiedlichen Ergebnissen führen je nachdem ob die zum GESIS Panel rekrutierten Menschen ohne Internet in die Analyse inkludiert oder exkludiert werden.

\section{Fallbeispiele: Das GIP und GESIS Panel}

Wie oben beschriebenen bestehen einige Forschungslücken in der Literatur zur Inklusion von Menschen ohne Internet in zufallsbasierte Onlinepanels. An dieser Stelle tragen wir zur Schließung dieser Lücken bei, indem wir einige weitere Erkenntnisse zu diesem Thema aus dem GIP und GESIS Panel präsentieren. Als bisher einzige Studie zeigen wir hier vergleichende Ergebnisse zu zwei unterschiedlichen Inklusionsstrategien (GIP: Ausstattung mit Geräten, GESIS Panel: postalische Befragungen als alternativer Teilnahme-Modus). Ebenso ist dies die 
bisher einzige Studie, die sowohl Ergebnisse zu Inklusionsstrategien in frisch rekrutierten Panels darstellt als auch im späteren regulären Panelbetrieb.

Für unsere Darstellung der Inklusionsstrategien im GIP und GESIS Panel beziehen wir alle Panel-Stichproben ein, die unabhängig voneinander gezogen wurden und einzig zu dem Zweck kontaktiert wurden, um das jeweilige Panel zu rekrutieren. Beim GIP handelt es sich dabei um zwei Stichproben: Das GIP 2012, welches die initiale Rekrutierung des GIP im Jahr 2012 beinhaltet, und das GIP 2014, welches eine unabhängige zusätzliche Rekrutierung des GIP darstellt, die im Jahr 2014 erfolgt ist. Eine weitere unabhängige Stichprobe des GIP, die 2018 rekrutiert wurde, wird nicht in die Darstellung einbezogen, weil sie mittels eines anderen Studiendesigns rekrutiert wurde. Beim GESIS Panel beziehen wir die initiale Stichprobe in unsere Darstellung ein, die 2013 rekrutiert wurde. Spätere Auffrischungs-Stichproben des GESIS Panels werden hier nicht berücksichtigt, da sie in anderer Form mittels eines anderen Studiendesigns rekrutiert wurden.

\section{Gemeinsamkeiten und Unterschiede im GIP und GESIS Panel}

Das GIP und GESIS Panel eignen sich in besonderer Weise für einen Vergleich der Inklusionsstrategien. Das liegt daran, dass sie viele Gemeinsamkeiten aufweisen, wie zum Beispiel der thematische Fokus, die Zielpopulation, der Rekrutierungszeitraum, und, vor allem, die Verwendung von Inklusionsstrategien für Menschen ohne Internet (siehe Tabelle 1 für einen Überblick über die wichtigsten Gemeinsamkeiten). 
Table 1: Gemeinsamkeiten des GIP und GESIS Panel

Inklusion von Menschen ohne Internet

Multithematische Panel-Befragungen mit sozialwissenschaftlichem Fokus

Zielpopulation: deutsche Bevölkerung

Ähnliche Rekrutierungszeiträume (zwischen 2012 und 2014)

Traditionelle mehrstufige zufallsbasierte Stichprobenziehungsverfahren

Persönliche Rekrutierungsbefragungen (face-to-face) und anschließende Panel-

Registrierungsbefragungen

Dasselbe Feldinstitut mit demselben Interviewer-Pool

Reguläre Panelbefragungen in einer Länge von 20 bis 25 Minuten alle zwei Monate

Während das GIP und GESIS Panel viele Gemeinsamkeiten aufweisen unterscheiden sie sich gleichzeitig in einer Reihe wichtiger Aspekte (für einen Überblick siehe Tabelle 2). 
Tabelle 2: Unterschiede zwischen GIP und GESIS Panel

\begin{tabular}{ll}
\hline German Internet Panel (GIP) & GESIS Panel \\
\hline Inklusion von Menschen ohne Internet per & Inklusion von Menschen ohne Internet \\
Ausstattung mit Geräten & mittels postalischer Befragungen \\
Stichprobenziehung mittels & Register-basierte Stichprobenziehung \\
Adressauflistungsverfahren (Personen sind & (Personen sind dadurch in Regionen \\
dadurch in Haushalten und Regionen & geklumpt) \\
geklumpt) & \\
Persönliche (face-to-face) & Persönliche (face-to-face) \\
Rekrutierungsinterviews mit einem & Rekrutierungsinterviews ausschließlich mit \\
beliebigen Haushaltsmitglied im Alter von & der aus dem Register gezogenen Person \\
mindestens 16 Jahren & \\
Alle Haushaltsmitglieder im Alter von & Ausschließlich die aus dem Register \\
mindestens 16 Jahren werden zum & gezogene Person wird zum Onlinepanel \\
Onlinepanel eingeladen & eingeladen \\
\hline
\end{tabular}

Die wichtigsten Unterschiede zwischen dem GIP und GESIS Panel lassen sich grob in zwei Kategorien zusammenfassen: Die Ausgestaltung der zufallsbasierten Stichprobenziehung und ihre Konsequenzen im weiteren Rekrutierungsprozess (d.h., wer wird initial kontaktiert, befragt und schließlich rekrutiert?) und die Strategie zur Inklusion von Menschen ohne Internet in das Onlinepanel. Die Unterschiede im Stichprobendesign und ihre Konsequenzen für die Rekrutierung sollen hier nicht weiter ausgeführt werden (für nähere Informationen hierzu siehe Blom et al., 2015, Blom et al., 2016 und Bosnjak et al. 2018). Stattdessen liegt der Fokus im Folgenden auf der Beschreibung der im GIP und GESIS Panel verwendeten 
Inklusionsstrategien und der anschießenden Darstellung einiger Erkenntnisse zu diesen Verfahren.

\section{Inklusionsstrategien im GIP und GESIS Panel}

Wie oben bereits angedeutet verwenden das GIP und GESIS Panel unterschiedliche Strategien zur Inklusion von Menschen ohne Internet. Im GIP werden alle Haushalte, die im Rekrutierungsinterview angeben, dass sie bisher nicht über eine (ausreichend schnelle) Internetverbindung und/oder ein funktionierendes internetfähiges Gerät verfügen mit entsprechender Ausrüstung ausgestattet. In der 2012 durchgeführten GIP-Rekrutierung bestand diese Ausrüstung aus einem Router zur WLAN-Internetverbindung und einem PC. In der 2014 durchgeführten GIP-Rekrutierung wurde statt eines PCs ein Tablet verwendet. In allen Fällen wurde die Installation der Geräte durch einen Dienstleister bei den betreffenden Haushalten vor Ort vorgenommen. Sobald die Installation durchgeführt war, wurde den betreffenden Personen außerdem eine Schulung im Umgang mit den Geräten durch den Dienstleister angeboten. Die Dienstleister waren speziell dazu ausgebildet, um den betreffenden Personen im GIP, die vor der Rekrutierung nicht mit dem Internet vertraut waren, die Nutzung der Geräte zum Ausfüllen der Befragungen beizubringen. Weiterhin waren die Geräte für eine vereinfachte Nutzung eingestellt. Das bedeutet zum Beispiel, dass standardmäßig ein großes Icon auf dem Startbildschirm vorinstalliert wurde, das die betreffenden Personen ohne Umschweife zum Login-Bereich der Studie führt, über den sie auf den Fragebogen zu der jeweils aktuellen Umfragewelle zugreifen können.

Das GESIS Panel dagegen verwendet eine andere Inklusionsstrategie, die darauf basiert Menschen ohne Internet per postalischer Befragung in das Panel einzubeziehen. Das bedeutet, dass allen Personen, die im Rekrutierungsinterview angeben, dass sie das Internet nicht für private Zwecke nutzen, angeboten wird per Papierfragebögen am Panel teilzunehmen. Zusätzlich geht das GESIS Panel in seinem Angebot über die postalische Befragung von 
Menschen ohne Internet hinaus. Es bietet nämlich auch internetnutzenden Personen, die einer Panelteilnahme per Internet skeptisch gegenüberstehen, die Möglichkeit, ebenfalls postalisch teilzunehmen. Damit geht das GESIS Panel in seiner Definition der Internetnutzung über das bloße Vorhandensein entsprechender notwendiger Ausrüstung hinaus und schafft ein Angebot auch an Menschen, die das Internet mit einer gewissen Skepsis oder nur zu bestimmten Zwecken nutzen.

Unabhängig von dem Grund, aus dem Personen den alternativen Befragungsmodus nutzen, werden ihnen die GESIS Panel-Fragebögen per Post mit frankiertem Rückumschlag zugesendet und werden von den entsprechenden Personen dann ausgefüllt per Post zurückgesendet.

\section{Erkenntnisse zur Inklusionsstrategie im GIP und GESIS Panel}

Im Vergleich der Inklusionsstrategien stellt sich zunächst die Frage, wie viele Personen mittels der im GIP und GESIS Panel implementierten Inklusionsstrategien zum Panel rekrutiert werden konnten. Insgesamt wurden im GIP 2012 und GIP 2014 5.004 Personen zum Panel rekrutiert (1.603 Personen in 2012 und 3.401 in 2014) und im GESIS Panel 4.938 Personen (siehe Tabelle $3)$. 
Tabelle 3. Übersicht über die Anzahl an rekrutierten Personen insgesamt und der per Inklusionsstrategie rekrutierten Personen sowie den Anteil der per Inklusionsstrategie rekrutierten Personen an der rekrutierten Panel-Stichprobe (eigene Berechnung).

\begin{tabular}{lccc} 
Panel & N Rekrutierte & Davon per Inklusionsstrategie & Anteil Inkludierte \\
\hline GIP (insgesamt) & 5.004 Personen & 364 Personen & $7,8 \%$ \\
GESIS Panel & 4.938 Personen & 1.865 Personen & $37,8 \%$ \\
(insgesamt) & & & \\
Ohne Internet & --- & 654 Personen & $13,2 \%$ \\
Mit Internet & --- & 1.211 Personen & $24,5 \%$
\end{tabular}

Im GIP wurden insgesamt 364 Personen mit Internet-Ausrüstung ausgestattet (110 davon bei der Rekrutierung in 2012 und 254 bei der Rekrutierung in 2014), was einem Anteil ausgestatteter Personen am Gesamt-Panel von 7,8\% entspricht. Im GESIS Panel dagegen wurden 1.865 Personen dem postalischen Befragungsmodus zugeordnet, was einem Anteil postalisch Befragter am Gesamt-Panel von 37,8\% entspricht. Damit ist der Anteil per Inklusionsstrategie in das Panel aufgenommener Personen im GESIS Panel deutlich höher als im GIP. Bei diesem Vergleich gilt es jedoch auch zu beachten, dass im GESIS Panel nicht nur Personen der postalische Modus angeboten wird, die das Internet nicht nutzen, sondern auch Personen, die das Internet nutzen aber nicht zur Teilnahme am Panel verwenden möchten. Wenn man in dieser Weise zwischen den postalisch Befragten im GESIS Panel differenziert, ergibt sich eine Anzahl von 654 Personen, die postalisch am Panel teilnehmen weil sie das Internet nicht nutzen (13,2\% des Gesamt-Panels) und 1.211 Personen, die postalisch befragt werden weil sie nicht im Internet an den Umfragen teilnehmen möchten (24,5\% des GesamtPanels).

Darüber hinaus ist es interessant zu sehen, inwiefern sich die beiden Panels darin unterscheiden Menschen, die das Internet nicht nutzen, in das Panel zu rekrutieren. Die oben zitierte Studie 
von Blom et al. (2015) zeigt für das GIP deutlich niedrigere Rekrutierungswahrscheinlichkeiten für Menschen ohne Internet (um 35\%-Punkte). Berechnungen für das GESIS Panel zeigen, dass die Rekrutierungswahrscheinlichkeit für Nicht-Internetnutzende ebenfalls niedriger ist, die Differenz mit 10\%-Punkten allerdings vergleichsweise gering ausfällt.

Während die Rekrutierung von Menschen ohne Internet zu einem Panel eine wichtige Voraussetzung dafür ist, dass solche Menschen in ein Onlinepanel inkludiert werden, so gibt die reine Anzahl an rekrutierten Menschen ohne Internet noch keinen Aufschluss darüber, ob diese Menschen im regulären Panelbetrieb nach der Rekrutierung auch an Panel-Befragungen teilnehmen. Tabelle 4 zeigt daher die Teilnahmeraten von Menschen, die per Inklusionsstrategie in das Onlinepanel rekrutiert wurden verglichen mit den anderen rekrutierten Panelteilnehmern exemplarisch für die jeweils 10. durchgeführte Befragungswelle im GIP und GESIS Panel. Die in 2012 und 2014 rekrutierten Stichproben des GIP werden für die folgenden Analysen zusammengenommen und ihre jeweilige 10. Befragungswelle verwendet. Die Teilnahmerate wird jeweils berechnet indem die Anzahl an Personen, die an der Befragungswelle teilgenommen hat durch die Anzahl an Personen geteilt wird, die zum Panel rekrutiert wurde. 
Tabelle 4. Teilnahmerate im GIP und GESIS Panel insgesamt sowie Teilnahmerate unter per Inklusionsstrategie Teilnehmender versus ohne Inklusionsstrategie Teilnehmender in der jeweiligen 10. regulären Panel-Befragungswelle.

\begin{tabular}{ll}
\hline Panel & Teilnahmerate \\
\hline GIP (insgesamt) & $65,0 \%$ \\
Befragte vormals ohne Internet & $64,6 \%$ \\
Befragte vormals bereits mit Internet & $65,1 \%$ \\
GESIS Panel (insgesamt) & $73,2 \%$ \\
Postalisch Befragte & $64,5 \%$ \\
Postalisch Befragte ohne Internet & $66,7 \%$ \\
Online Befragte & $78,5 \%$ \\
\end{tabular}

Insgesamt haben an der 10. Befragungswelle im GIP 65,0\% der rekrutierten Personen teilgenommen. Unter denjenigen, die mit Ausrüstungsgegenständen ausgestattet wurden („Befragte vormals ohne Internet“) lag die Teilnahmerate bei 64,6\% und unter denjenigen, die bereits entsprechende Ausrüstung besaßen lag die Teilnahmerate bei 65,1\%. Im GESIS Panel haben an der 10. Befragungswelle 73,2\% der rekrutierten Personen teilgenommen. Unter denjenigen, die postalisch befragt wurden, lag die Teilnahmerate bei 64,5\% und bei denjenigen, die per Internet befragt wurden lag die Teilnahmerate bei 78,5\%. Wenn man im GESIS Panel nur diejenigen Personen betrachtet, die postalisch befragt werden, weil sie das Internet nicht nutzen, so liegt deren Teilnahmerate bei $66,7 \%$.

Wenn man die Teilnahmeraten innerhalb der Panels vergleicht, so kann gesagt werden, dass es im GIP im Wesentlichen keinen Unterschied in den Teilnahmeraten von Menschen gibt, die mit Ausrüstung ausgestattet wurden, und Menschen, die bereits vor der Rekrutierung Internetzugang besaßen. Im GESIS Panel dagegen liegt die Teilnahmerate von Personen, die 
postalisch befragt werden unter der Teilnahmerate von Personen, die per Internet befragt werden. Die Differenz ist insbesondere groß, wenn man nicht nur diejenigen Personen betrachtet, die postalisch am GESIS Panel teilnehmen, weil sie kein Internet haben, sondern auch die Personen miteinbezieht, die postalisch teilnehmen, weil sie zwar Internetzugang haben, diesen aber nicht zur Teilnahme am GESIS Panel nutzen wollen. Diese Differenz in den Teilnahmeraten ist möglicherweise ein Modus-Effekt. Dieser wird eventuell dadurch verstärkt, dass Personen, die per Internet am GESIS Panel teilnehmen, eine Erinnerungs-Email erhalten, wenn sie noch nicht an einer Befragungswelle teilgenommen haben, während kein äquivalentes Erinnerungsschreiben an Personen versendet wird, die postalisch am GESIS Panel teilnehmen. Da die am GESIS Panel teilnehmenden Menschen nicht zufällig auf die beiden Modus-Gruppen verteilt werden, sondern sich selbst in den jeweiligen Modus selektieren, ist diese Erklärung allerdings als spekulativ anzusehen.

Zuletzt soll hier noch zur Beantwortung der Frage beigetragen werden, ob die jeweiligen Inklusionsstrategien zur Verbesserung der Stichprobenqualität der jeweiligen Onlinepanels beitragen. Diese Frage ist für die jeweils frisch rekrutierte Panel-Stichprobe des GIP und GESIS Panel bereits an anderer Stelle positiv beantwortet worden (siehe Blom et al., 2017, Bosnjak, 2018 und den Literaturüberblick oben). An dieser Stelle präsentieren wir daher zusätzliche Erkenntnisse dazu, ob diese Verbesserung der Stichprobenqualität auch im regulären Panelbetrieb nach der Rekrutierung Bestand hat. Tabelle 5 zeigt entsprechende Ergebnisse im Durchschnitt über die jeweils ersten zwölf Befragungswellen des GIP und GESIS Panel. 
Tabelle 5. Vergleich der Befragten-Stichproben des GIP und GESIS Panel (aggregiert über die ersten zwölf regulären Befragungswellen) mit der offiziellen Bevölkerungsstatistik aus dem Mikrozensus (MZ) ${ }^{1}$.

\begin{tabular}{lcccccc}
\hline Merkmal & MZ & GIP & $\begin{array}{c}\text { Ohne } \\
\text { Inklusion }\end{array}$ & $\begin{array}{c}\text { GESIS } \\
\text { Panel }\end{array}$ & $\begin{array}{c}\text { Ohne } \\
\text { Inklusion }\end{array}$ & $\begin{array}{c}\text { Ohne } \\
\text { Inklusion } \\
\text { Internetloser }\end{array}$ \\
\hline Geschlecht & & & & & & \\
Weiblich & $50,0 \%$ & $49,8 \%$ & $49,1 \%$ & $52,2 \%$ & $49,9 \%$ & $51,7 \%$ \\
Männlich & $50,0 \%$ & $50,2 \%$ & $50,9 \%$ & $47,8 \%$ & $50,1 \%$ & $48,3 \%$ \\
Alter & & & & & & \\
18 bis 30 & $20,6 \%$ & $21,1 \%$ & $21,9 \%$ & $18,0 \%$ & $23,1 \%$ & $20,1 \%$ \\
31 bis 40 & $17,5 \%$ & $15,9 \%$ & $16,4 \%$ & $14,9 \%$ & $17,7 \%$ & $16,5 \%$ \\
41 bis 50 & $22,9 \%$ & $23,0 \%$ & $23,2 \%$ & $24,5 \%$ & $25,1 \%$ & $25,8 \%$ \\
51 bis 60 & $21,7 \%$ & $24,2 \%$ & $23,9 \%$ & $23,9 \%$ & $20,8 \%$ & $22,6 \%$ \\
61 bis 70 & $17,3 \%$ & $15,8 \%$ & $14,7 \%$ & $18,7 \%$ & $13,3 \%$ & $14,9 \%$ \\
Bildung & & & & & & \\
Gering & $32,4 \%$ & $18,8 \%$ & $17,8 \%$ & $22,9 \%$ & $13,6 \%$ & $17,9 \%$ \\
Mittel & $34,3 \%$ & $33,7 \%$ & $33,0 \%$ & $34,3 \%$ & $33,6 \%$ & $35,1 \%$ \\
Hoch & $33,3 \%$ & $47,6 \%$ & $49,2 \%$ & $42,8 \%$ & $52,7 \%$ & $47,0 \%$ \\
\hline
\end{tabular}

Insgesamt kann für das GIP festgestellt werden, dass im Durchschnitt über die ersten zwölf Befragungswellen die Inklusion von Menschen ohne Internet dabei hilft die Unterrepräsentierung von Frauen, älteren Menschen (61 bis 70 Jahre) und gering Gebildeten (d.h. Personen ohne oder mit geringem Schulabschluss) im Vergleich zur offiziellen Bevölkerungsstatistik zu verringern. Das Geschlecht betreffend erhöht die Inklusionsstrategie den Frauenanteil im Durchschnitt von 49,1\% auf 49,8\% (verglichen mit 50,0\% in der offiziellen Statistik). In Bezug auf das Alter erhöht die Inklusion von Menschen ohne Internet den Anteil älterer Menschen im Durchschnitt von 14,7\% auf 15,8\% im GIP (verglichen mit 17,3\% in der offiziellen Statistik). Im Hinblick auf die Bildung erhöht die Inklusion von Menschen ohne

\footnotetext{
${ }^{1}$ siehe https://www.destatis.de/DE/Themen/Gesellschaft-Umwelt/Bevoelkerung/HaushalteFamilien/Methoden/mikrozensus.html
} 
Internet den Anteil gering Gebildeter von 17,8\% auf 18,8\% (verglichen mit 32,4\% in der offiziellen Statistik).

Im GESIS Panel hilft die Inklusion von Menschen ohne Internet im Durchschnitt über die ersten zwölf Befragungswellen nicht dabei die Repräsentativität im Hinblick auf das Geschlecht zu erhöhen. In der Tendenz ist es eher umgekehrt. Während der Frauenanteil im Durchschnitt bei 49,9\% liegt wenn man die postalisch befragten Personen nicht berücksichtigt (was fast exakt dem Frauenanteil in der offiziellen Statistik von 50,0\% entspricht), so steigt der Frauenanteil unter Einbezug der postalisch befragten Personen auf 52,2\%, was leicht über dem Anteil in der offiziellen Statistik liegt. Beim Alter und der Bildung hingegen hat die Inklusionsstrategie des GESIS Panel einen positiven Effekt. So steigt der Anteil älterer Menschen durch die Inklusion postalisch Befragter von 13,3\% auf 18,7\% (verglichen mit 17,3\% in der offiziellen Statistik) und der Anteil gering Gebildeter steigt von 13,6\% auf 22,9\% (verglichen mit 32,4\% in der offiziellen Statistik).

Wenn man die durchschnittliche Befragtenstichprobe im GESIS Panel insgesamt mit derjenigen vergleicht, die nur die Menschen ausschließt, die nicht das Internet nutzen (im Gegensatz zu denen, die das Internet nutzen, aber nicht für die Panel-Befragungen nutzen wollen) so ergeben sich ähnliche Erkenntnisse wie im Hinblick auf die Stichprobe, die alle postalisch Befragten ausschließt. Der Effekt ist allerdings insgesamt geringer. So wird der Anteil der Frauen, älteren Menschen, und gering Gebildeten durch Inklusion nur derer, die tatsächlich nicht das Internet nutzen im GESIS Panel in geringerem Maße erhöht als wenn auch Personen, die ihren Internetzugang nicht für das GESIS Panel nutzen wollen einbezogen werden. In Bezug auf die Bildung bedeutet das, dass es sich für das GESIS Panel in Bezug auf die Stichprobenqualität auszahlt den alternativen Befragungsmodus nicht nur denjenigen Menschen anzubieten, die das Internet nicht nutzen, sondern auch solchen Menschen, die das Internet zwar nutzen, es aber nicht zur Teilnahme am GESIS Panel verwenden wollen. 


\section{Schlussfolgerungen und Ausblick}

Der vorliegende Beitrag beschäftigt sich mit der Inklusion von Menschen ohne Internet in zufallsbasierte Onlinepanels. Dieses Thema ist deshalb relevant, weil sich Menschen ohne Internet systematisch von Menschen mit Internet unterscheiden, zum Beispiel in Alter und Bildung, aber auch in Folge infrastruktureller Benachteiligung von Menschen, die in bestimmten ländlichen Regionen leben. Insofern die Exklusion dieser Bevölkerungsgruppen potentiell die Befragungsergebnisse verfälschen kann, nutzen eine Reihe etablierter Onlinepanels Strategien zur Inklusion von Menschen ohne Internet. Diese Strategien lassen sich in zwei Kategorien zusammenfassen: die Ausstattung von Menschen ohne Internet mit entsprechender Ausrüstung (z.B. WLAN und Tablets) zur Teilnahme an Online-Befragungen und das Anbieten eines alternativen Befragungsmodus (i.d.R. postalisch oder telefonisch).

Im vorliegenden Beitrag wurden die in der Praxis verwendeten Inklusionsstrategien beschrieben und die wenigen Studien zu deren Erfolg diskutiert. Der Überblick über die vorhandene Forschungsliteratur macht deutlich, dass es zu vielen Aspekten der Inklusionsstrategien wenig bis keine empirischen Erkenntnisse gibt. Dies betrifft unter anderem die Frage welchen Effekt die Inklusion von Menschen ohne Internet über den Zeitpunkt der Onlinepanel-Rekrutierung hinaus hat (also im regulären Panelbetrieb) und auch die Frage, ob unterschiedliche Inklusionsstrategien divergierende Effekte auf die Onlinepanels haben.

In einer Fallstudie haben wir daher einige Erkenntnisse aus dem GIP und GESIS Panel zu diesen Fragen präsentiert. Diese beiden zufallsbasierten Onlinepanels nutzen unterschiedliche Ansätze der Inklusion (GIP: Ausstattung mit Internet, GESIS Panel: alternativer Befragungsmodus). Die präsentierten Ergebnisse legen nahe, dass die Inklusion von Menschen ohne Internet auch im regulären Panelbetrieb einen positiven Einfluss auf die Stichprobenqualität hat, insbesondere im Hinblick auf die Bildung. Weiterhin deuten die Ergebnisse darauf hin, dass der Effekt mittels der Ausstattung mit Internet im GIP einerseits 
zwar mögliche Modus-Effekte vermeidet (z.B. im Hinblick auf das Teilnahme-Verhalten), andererseits aber auch zu einer geringeren Repräsentativitätssteigerung in Bezug auf die Bildung führt als dies im GESIS Panel mittels alternativem Befragungsmodus der Fall ist.

Insgesamt ist die in diesem Beitrag präsentierte Fallstudie ein erster Schritt, um die Auswirkungen der in der Praxis verwendeten Inklusionsstrategien zu verstehen. Um gesicherte Aussagen über die Auswirkungen verschiedener Inklusionsstrategien in Bezug auf Rekrutierungserfolg, Panelteilnahme und Stichprobenqualität zu treffen bedarf es weiterer Forschung. Insbesondere wäre ein vollständig experimentelles Design, in dem Befragte zufällig einer der möglichen Inklusionsstrategien zugeordnet werden, notwendig um die Strategien in Bezug auf ihre Kosten und Nutzen vergleichen zu können. Dabei wäre es sinnvoll, neben Aspekten der Rekrutierung, Panelteilnahme und Stichprobenqualität auch mögliche Messfehler zu untersuchen, die zum Beispiel durch mangelnde Beherrschung der Technik im Falle der Ausstattungsstrategie oder Messunterschiede pro Befragungsmodus in der Strategie des Anbietens eines alternativen Modus betreffen.

Darüber hinaus wäre es wichtig, den Fortschritt der Digitalisierung konzeptuell mit zu denken, um passende Inklusionsstrategien auch in Zukunft anbieten zu können. Dies betrifft zum einen die mögliche Notwendigkeit, den Begriff der Internetnutzung neu zu denken. Dabei könnte es sinnvoll sein, nicht mehr binär zwischen Menschen zu unterscheiden, die das Internet gar nicht nutzen und Menschen, die das Internet grundsätzlich nutzen. Die beschriebene Tatsache, dass im Rahmen des GESIS Panels ein nicht unerheblicher Anteil von Personen, die das Internet nutzen, dennoch postalisch teilnehmen, gibt erste Hinweise darauf, dass eine binäre Nutzungsdefinition zu kurz greift, wie es auch in der allgemeinen Forschung zur Internetnutzung diskutiert wird (siehe bspw. Blank \& Groselj, 2014). Für das GIP zeigen Herzing und Blom (2018), dass die Teilnahmewahrscheinlichkeit mit der digitalen Affinität steigt. Es geht also nicht allein darum, ob eine Person das Internet nutzt, sondern in erster Linie 
darum, wie sie es nutzt und wie vertraut sie mit verschiedenen Aspekten ist, die auch eine erfolgreiche Teilnahme an einer Onlinebefragung begünstigt.

Bei der Weiterentwicklung der Definition von Internetnutzung sind in besonderem Maße auch Aspekte des mobilen Zugangs zu bedenken. Die Hürde an einer kurzen Befragung per Internet teilzunehmen ist zum Beispiel geringer für Menschen, die zu jedem Zeitpunkt per Smartphone schnell auf das Internet zugreifen können als bei Personen, die dies nur stationär in ihrer Wohnung tun können nachdem sie den PC hochgefahren haben. Die Mehrheit der im vorliegenden Beitrag genannten zufallsbasierten Onlinepanels wurden zu einem Zeitpunkt konzipiert, als die mobile Internetnutzung noch nicht so weit verbreitet war wie gegenwärtig. So nimmt der Anteil derjenigen Personen, die an Online-Umfragen mit einem mobilen Endgerät teilnehmen stetig zu (siehe bspw. Weiß et al., 2019) und der Anteil der Personen, die ausschließlich mit einem mobilen Endgerät das Internet nutzen steigt ebenfalls zunehmend. Diese Personen unterscheiden sich wiederum von der Gesamtbevölkerung und sind in der Tendenz jünger und niedriger gebildet (Antoun, 2015). In Zukunft könnten daher die aus der Literatur bekannte Verzerrungen in den Onlinepanel-Stichproben (z.B. Unterrepräsentierung von gering Gebildeten) durch mobile Internetnutzende ausgeglichen werden. Dazu wird es eine wichtige Herausforderung der zufallsbasierten Onlinepanels sein, Strategien zu entwickeln, um Befragungen so zu konzipieren, dass auch Personen, die präferiert oder sogar ausschließlich mit mobilen Endgeräten an die Befragungen teilnehmen würden, in das Panel zu rekrutieren und dauerhaft im Panel zu halten. 


\section{Referenzen}

Antoun, C. 2015. Who Are the Internet Users, Mobile Internet Users, and Mobile-Mostly Internet Users?: Demographic Differences across Internet-Use Subgroups in the U.S.. In: Toninelli, D, Pinter, R \& de Pedraza, P (eds.) Mobile Research Methods: Opportunities and Challenges of Mobile Research Methodologies , Pp. 99-117. London: Ubiquity Press.

Blank, G., \& Groselj, D. (2014). Dimensions of Internet use: amount, variety, and types. Information, Communication \& Society, 17(4), 417-435.

Blom, A. G., Cornesse, C., Friedel, S., Krieger, U., Fikel, M., Rettig, T., Wenz, A., Juhl, S., Lehrer, R., Möhring, K., Naumann, E. \& Reifenscheid, M. (2020). High Frequency and High Quality Survey Data Collection. Survey Research Methods 14(2), 171-178.

Blom, A. G., Herzing, J. M., Cornesse, C., Sakshaug, J. W., Krieger, U., \& Bossert, D. (2017). Does the recruitment of offline households increase the sample representativeness of probability-based online panels? Evidence from the German internet panel. Social Science Computer Review 35(4), 498-520.

Blom, A. G., Bosnjak, M., Cornilleau, A., Cousteaux, A. S., Das, M., Douhou, S., \& Krieger, U. (2016). A comparison of four probability-based online and mixed-mode panels in Europe. Social Science Computer Review 34(1), 8-25.

Blom, A. G., Gathmann, C., \& Krieger, U. (2015). Setting up an online panel representative of the general population: The German Internet Panel. Field methods 27(4), 391-408.

Bosnjak, M., Dannwolf, T., Enderle, T., Schaurer, I., Struminskaya, B., Tanner, A., \& Weyandt, K. W. (2018). Establishing an open probability-based mixed-mode panel of the general population in Germany: The GESIS panel. Social Science Computer Review 36(1), 103-115. 
Bosnjak, M., Haas, I., Galesic, M., Kaczmirek, L., Bandilla, W., \& Couper, M. P. (2013). Sample Composition Discrepancies in Different Stages of a Probability-based Online Panel. Field Methods 25(4), 339-360.

Bundesministerium für Verkehr und digitale Infrastruktur (2020). Der Breitbandatlas. https://www.bmvi.de/DE/Themen/Digitales/Breitbandausbau/Breitbandatlas-Karte/start.html. Zugegriffen: 29. Januar 2020.

Callegaro, M., Baker, R., Bethlehem, J. G., Göritz, A. S., Krosnick, J. A., \& Lavrakas, P. J. (2014). Online panel research. History, concepts, aapplication and a look at the future. In M. Callegaro, R. Baker, J. Bethlehem, A. S. GÃđIritz, J. A. Krosnick, \& P. J. Lavrakas (Hrsg.), Online Panel Research. A Data Quality Perspective (S. 1-22). Wiley.

CentERdata (2020). LISS Panel. https://www.lissdata.nl/. Zugegriffen: 10.06.2020.

Center for Asian Public Opinion Research \& Collaboration Initiative (2020). KAMOS. http://www.cnukamos.com/eng/main/. Zugegriffen: 10.06.2020.

Cornesse, C., Blom, A. G., Dutwin, D., Krosnick, J. A., De Leeuw, E. D., Legleye, S., Pasek, J., Pennay, D., Phillips, B., Sakshaug J. W., Struminskaya, B., \& Wenz, A. (2020). A Review of Conceptual Approaches and Empirical Evidence on Probability and Nonprobability Sample Survey Research. Journal of Survey Statistics and Methodology 8(1), 4-36.

Destatis (2020). Durchschnittliche Nutzung des Internets durch Personen nach Altersgruppen. https://www.destatis.de/DE/Themen/Gesellschaft-Umwelt/Einkommen-KonsumLebensbedingungen/IT-Nutzung/Tabellen/durchschnittl-nutzung-alter-ikt.html. Zugegriffen: 28. Januar 2020.

DiSogra, C., \& Callegaro, M. (2016). Metrics and Design Tool for Building and Evaluating Probability-Based Online Panels. Social Science Computer Review, 34(1), 26-40. 
Eckman, S. (2016). Does the Inclusion of Non-Internet Households in a Web Panel Reduce Coverage Bias? Social Science Computer Review 34(1), 41-58.

Eynon, R., \& Helsper, E. (2011). Adults learning online: Digital choice and/or digital exclusion? New Media \& Society, 13(4), 534-551.

GALLUP (2020). Gallup Panel. https://www.gallup.com/analytics/213695/gallup-panel.aspx. Zugegriffen: 10.06.2020.

Heerwegh, D. (2009). Mode differences between face-to-face and web surveys: an experimental investigation of data quality and social desirability effects. International Journal of Public Opinion Research 21(1), 111-121.

Helsper, E. J., \& Reisdorf, B. C. (2017). The emergence of a "digital underclass" in Great Britain and Sweden: Changing reasons for digital exclusion. New Media \& Society, 19(8), $1253-1270$.

Herzing, J. M. E., \& Blom, A. G. (2018). The Influence of a Person's Digital Affinity on Unit Nonresponse and Attrition in an Online Panel. Social Science Computer Review, 089443931877475 .

Internet World Stats. Internet Usage in the European Union. https://www.internetworldstats.com/stats9.htm. Zugegriffen: 28. Januar 2020.

Ipsos (2020). KnowledgePanel. https://www.ipsos.com/en-us/solutions/publicaffairs/knowledgepanel. Zugegriffen: 10.06.2020.

Jessop, Curtis. 2017. „Developing the NatCen Panel“. http://www.natcen.ac.uk/media/1484228/Developing-the-NatCen-Panel-V2.pdf. Zugegriffen 27. Mai 2019. 
König, R., Seifert, A., \& Doh, M. (2018). Internet use among older Europeans: an analysis based on SHARE data. Universal Access in the Information Society 17(3), 621-633.

Leenheer, J., \& Scherpenzeel, A. C. (2013). Does it pay off to include non-Internet households in an Internet panel? International Journal of Internet Science 8(1), 17-28.

NatCen Social Research (2020). NatCen Panel. http://www.natcen.ac.uk/taking-part/studiesin-field/natcen-panel/. Zugegriffen 10. Juni 2020.

NORC (2019). Technical Overview of the AmeriSpeak Panel. NORC's Probability-Based Household

Panel. https://amerispeak.norc.org/Documents/Research/AmeriSpeak\%20Technical\%20Overview\% 202019\%2002\%2018.pdf. Zugegriffen: 09. Juni 2020.

Pagel, L., \& Schupp, J. (2019). Die Haushaltspanelstudie sozio-ökonomisches Panel (SOEP) und ihre Potenziale für Sekundäranalysen. In: M.-C. Begemann und K. Birkelbach (Hrsg.). Forschungsdaten für die Kinder- und Jugendhilfe (S.165-186). Springer VS, Wiesbaden.

Pew Research Center (2019). Growing and Improving Pew Research Center's American Trends Panel. https://www.pewresearch.org/methods/2019/02/27/growing-and-improvingpew-research-centers-american-trends-panel/. Zugegriffen: 09.06.2020.

Pforr, K., \& Dannwolf, T. (2017). What do we lose with online-only surveys? Estimating the bias in selected political variables due to online mode restriction. Statistics, Politics and Policy $8(1), 105-120$.

Prensky, M. (2001). Digital Natives, Digital Immigrants. On the Horizon 9 (5): 1-6.

Probit Inc (2020). Probit Panel. https://probit.ca/. Zugegriffen: 10. Juni 2020. 
Revilla, M., Cornilleau, A., Cousteaux, A.-S., Legleye, S., \& de Pedraza, P. (2016). What Is the Gain in a Probability-Based Online Panel of Providing Internet Access to Sampling Units Who Previously Had No Access? Social Science Computer Review 34(4), 479-496.

Rookey, B. D., Hanway, S., \& Dillman, D. A. (2008). Does a Probability-Based Household Panel Benefit from Assignment to Postal Response as an Alternative to Internet-Only? Public Opinion Quarterly 72(5), 962-984.

Schaurer, I., \& Weiß, B. (2020). Investigating selection bias of online surveys on coronavirusrelated behavioral outcomes. Survey Research Methods 14(2), 103-108.

Schleife, K. (2010). What really matters: Regional versus individual determinants of the digital divide in Germany. Research Policy, 39(1), 173-185. https://doi.org/10.1016/j.respol.2009.11.003. Zugegriffen: 12. Juni 2020.

Seifert, A., \& Schelling, H. R. (2018). Seniors online: Attitudes toward the internet and coping with everyday life. Journal of Applied Gerontology 37(1), 99-109.

Statista (2020). Global digital population as of April 2020. https://www.statista.com/statistics/617136/digital-population-worldwide/. Zugegriffen: 09. Juni 2020.

Toepoel, V. (2016). The Impact of Non-Coverage in Web Surveys in a Country with High Internet Penetration: Is It (Still) Useful to Provide Equipment to Non-Internet Households in the Netherlands? International Journal of Internet Science 11(1), 33-50.

Toepoel, V., \& Hendriks, Y. (2016). The Impact of Non-Coverage in Web Surveys in a Country with High Internet Penetration: Is It (Still) Useful to Provide Equipment to Non-Internet Households in the Netherlands? 11(1), 33-50. 
Van Laar, E., Van Deursen, A. J., Van Dijk, J. A., \& De Haan, J. (2017). The relation between 21st-century skills and digital skills: A systematic literature review. Computers in human behavior 72, 577-588.

Universität Mannheim (2020). German Internet Panel. https://www.uni-mannheim.de/gip/. Zugegriffen: 10. Juni 2020.

University of Southern California (2017). Understanding America Study. https://uasdata.usc.edu/index.php. Zugegriffen: 10. Juni 2020.

Vannieuwenhuyze, J. T., \& Loosveldt, G. (2013). Evaluating relative mode effects in mixedmode surveys: three methods to disentangle selection and measurement effects. Sociological Methods \& Research 42(1), 82-104.

Wasmer, M., Blohm, M., Walter, J., Jutz, R., Scholz, E. (2017). Konzeption und Durchführung der „Allgemeinen Bevölkerungsumfrage der Sozialwissenschaften (ALLBUS) 2014. GESIS Paper 2017 | 20. https://www.gesis.org/en/allbus/contents-search/methodological-reports. Zugegriffen: 09. Juni 2020.

Weiß, Bernd, Henning Silber, Bella Struminskaya, and Gabriele Durrant. 2019. "Mobile Befragungen." In Handbuch Methoden der empirischen Sozialforschung, edited by Baur Nina, and Blasius Jörg, 801-812. Wiesbaden: Springer Fachmedien. 


\section{Kontaktinformationen der Autorinnen}

Dr. Carina Cornesse

Universität Mannheim

B6, 30-32, 68131 Mannheim

carina.cornesse@uni-mannheim.de

Dr. Ines Schaurer

GESIS - Leibniz Institut für Sozialwissenschaften

B2,1

68159 Mannheim

ines.schaurer@gesis.org

\section{Danksagung}

Der vorliegende Beitrag verwendet Daten des German Internet Panel (GIP) und GESIS Panel. Das GIP wird über den Sonderforschungsbereich (SFB) 884 „Politische Ökonomie der Reformen“ über die Deutsche Forschungsgemeinschaft (DFG) finanziert (Projekt-ID: 139943784). Das GESIS Panel wird von GESIS - Leibniz Institut for Sozialwissenschaften finanziert. Die Autorinnen danken dem SFB 884, besonders den Projekten A8 und Z1, sowie GESIS für die Unterstützung. Die Autorinnen danken außerdem Elena Madiai für ihre Hilfe bei der Manuskriptgestaltung. 